\title{
An Optimal Electric Dipole Antenna Model and Its Field Propagation
}

\author{
Yidong Xu, ${ }^{1}$ Lili Guo, ${ }^{1}$ Wei Xue, ${ }^{1}$ Korochentsev Vladimir, ${ }^{2}$ and Junwei Qi ${ }^{1}$ \\ ${ }^{1}$ College of Information and Communication Engineering, Harbin Engineering University, Harbin 150001, China \\ ${ }^{2}$ School of Engineering, Far Eastern Federal University, Vladivostok 690950, Russia \\ Correspondence should be addressed to Wei Xue; xuewei@hrbeu.edu.cn
}

Received 4 February 2016; Revised 5 March 2016; Accepted 17 April 2016

Academic Editor: Christoph F. Mecklenbräuker

Copyright (c) 2016 Yidong Xu et al. This is an open access article distributed under the Creative Commons Attribution License, which permits unrestricted use, distribution, and reproduction in any medium, provided the original work is properly cited.

\begin{abstract}
An optimal electric dipole antennas model is presented and analyzed, based on the hemispherical grounding equivalent model and the superposition principle. The paper also presents a full-wave electromagnetic simulation for the electromagnetic field propagation in layered conducting medium, which is excited by the horizontal electric dipole antennas. Optimum frequency for field transmission in different depth is carried out and verified by the experimental results in comparison with previously reported simulation over a digital wireless Through-The-Earth communication system. The experimental results demonstrate that the dipole antenna grounding impedance and the output power can be efficiently reduced by using the optimal electric dipole antenna model and operating at the optimum frequency in a vertical transmission depth up to $300 \mathrm{~m}$ beneath the surface of the earth.
\end{abstract}

\section{Introduction}

Electric dipole antennas have been widely used in ThroughThe-Earth (TTE) communication via a current injected into the earth at the power stage [1-3]. The Coal Mine Security Standard has many restrictions on the permissible transmission power of TTE communication systems. There are many methods which have been proposed and investigated to reduce the output power by changing the shape and material, increasing the interelectrode distance $[4,5]$, or using only one electrode with different kinds of material, such as parabolt, copper braid extended aluminum foil, and steel rod [3]. However, these methods have not discussed the grounding impedance model of several electrodes in-array and still cannot reduce the electrodes grounding impedance to a level less than $300 \Omega$. Many reports take the contact impedance between the electrode and the earth into account [2] and suggest lowering it with resistance reducing material but have not given the quantitative relationship between them. Some models have been proposed and investigated the dipole antenna in two- or three-layered conducting medium [68]. However, most of them discuss the propagation from surface to surface or for far field transmission and have not given the optimal operating frequency to reduce the TTE communication power in a stratified dissipative medium vertically. In [9], the author gives the electromagnetic wave radiation propagate in four-layered medium model in the VHF/UHF bands which is a great limitation for TTE communication. References $[7,10]$ have proposed the models of extremely low frequency electromagnetic field generated by submerged horizontal electric dipole (HED) in three-layer medium, where the field point and the source point are in the same layer. These models cannot be applied in practical TTE communication because the source point and the field point are not in the same layer.

To reduce the output power, a model for the parallel grounding impedance of several HED antennas arrayed inline is presented and the quantitative relationship between the resistance reducing material and the impedance is proposed in this paper. It is found that the grounding impedance model of several electrodes in-array has good match with the experimental results, and the optimal electric dipole antenna can reduce the grounding impedance to less than $5 \Omega$. In order to further lower the output power and to improve communication quality, a full-wave electromagnetic simulation model for the electromagnetic field propagation in layered conducting medium is proposed, which is excited by the HED antennas. An optimum frequency for field 
transmission at different propagation depth is carried out by this model. The receiver can get a maximum field when the TTE communication transmitter works at this optimum frequency, which is numerically and experimentally verified over a digital wireless system.

\section{Modeling and Optimization of an Electrical Dipole Antenna}

2.1. HED Antenna Grounding Model. Electric dipole antennas are used to inject electrical power into the earth by establishing galvanic coupling between the earth and the power stage of the transmitter. The frequency-dependent grounding impedance includes the wire impedance, the contact impedance between the wire and the electrode, the impedance of the electrode, the earth current divergence impedance, and the contact impedance between the earth and the electrode $[2,11,12]$. However, when the grounding impedance is in the frequency range of lower than $50 \mathrm{kHz}$, the grounding electrode exhibits the grounding impedance close to the grounding resistance [13]. In this paper, we investigate the earth current divergence impedance.

For a horizontal grounding electrode, with a length $L$, a diameter $d$, and an embedded depth $h$, six possible earth current divergence impedance models are presented in Table 1 [12].

For the sake of simplicity, the resistance of a horizontal grounding electrode with an embedded depth of zero can be expressed by the neutral-point potential method. This states that increasing the earth conductivity and the size of electrode can reduce its current divergence resistance. By placing several electrodes in parallel as one new electrode, the grounding impedance can be further reduced. The new grounding impedance $R_{d}$ can be expressed as follows:

$$
R_{d}=\frac{R_{L}}{n \eta}
$$

where $R_{L}$ is the grounding impedance of a single electrode; $n$ is the number of electrodes; $\eta$ is the grounding electrode utilization factor, which is determined by the shape and number of electrodes and their relative positions.

The parallel resistance $R_{d}$ can be similarly calculated by introducing a hemispherical grounding equivalent model [12]. For a horizontal grounding electrode buried at the subsurface level, its equivalent radius expression can be obtained based on image theory and the neutral-point potential method as

$$
r=\frac{L}{2 \ln (2 L / d)}
$$

For $n$ parallel horizontal grounding electrodes arrayed in-line, the distance between two adjacent electrodes is $D$. In order to simplify the calculation, a radius ratio $\alpha=$ $r / D$ is defined. The injection current over each electrode is $\left\{I_{1}, I_{2}, \ldots, I_{i}, \ldots, I_{n}\right\}$ with the the electric potential $V$. The electric potential of the $i$ th electrode as a reference electrode is given by $\Phi_{i}=(1 / \sigma)(1 / 2 \pi r) I_{i}$, based on the hemispherical grounding equivalent model. The electric potential provided by the $i \pm k$ th electrode at the $i$ th electrode can be expressed as $\Phi_{i \pm k}=(1 / \sigma)(1 / 2 \pi r)(\alpha / k) I_{i \pm k}$. Then, we obtain the electric potential $V=\sum_{i=1}^{n} \Phi_{i}$ by the superposition principle. By setting each electrode as reference, we have

$$
\begin{aligned}
& \frac{1}{\sigma} \frac{1}{2 \pi r}\left[\begin{array}{ccccc}
1 & \alpha & \frac{\alpha}{2} & \cdots & \frac{\alpha}{n-1} \\
\alpha & 1 & \alpha & \cdots & \frac{\alpha}{n-2} \\
\frac{\alpha}{i-1} & \cdots & 1 & \cdots & \frac{\alpha}{n-i} \\
\frac{\alpha}{n-1} & \frac{\alpha}{n-2} & \frac{\alpha}{n-3} & \cdots & 1
\end{array}\right]\left[\begin{array}{c}
I_{1} \\
I_{2} \\
\vdots \\
I_{i} \\
\vdots \\
I_{n}
\end{array}\right] \\
& =\left[\begin{array}{c}
V \\
V \\
\vdots \\
V \\
\vdots \\
V
\end{array}\right] \\
& R_{d}=\frac{V}{\sum_{i=1}^{n} I_{i}}=\frac{R_{L}}{n \eta} \\
& \alpha=\frac{r}{D} \text {. }
\end{aligned}
$$

When $n=\{2,3,4,5,6\}$, the expressions of grounding electrode utilization factor $\eta$ with argument radius ratio $\alpha$ are obtained and shown in Table 2 . The variations of $\eta$ with $\alpha^{-1}$ are shown in Figure 1.

From Figure 1, it can be seen that the grounding electrode utilization factor $\eta$ decreases as the parameter $\alpha$ increases for the same number of electrodes $n$. The main reason is that the shielding effect increases as the contiguous electrodes distance decreases, restricting the current injection into the earth. When there is no shielding effect between electrodes, $\alpha$ becomes infinitely small and $\eta$ tends towards 1 .

The grounding impedance can be reduced when the grounding electrodes are surrounded by high conductivity resistance reducing material [14].

Semicylinder pits with length $L$ and radius $b$, where $b \ll L$, are filled with resistance reducing material with conductivity $\sigma_{s}$. Metal horizontal grounding electrodes with length $L$ and radius $a=d / 2$ are buried at the pit center. The transitional area is the area between the resistance reducing material and the surrounding soil which depends on the seepage property of the material, and it expands the pits to an equivalent radius $b_{m}$. The soil moisture, temperature, and soil gap have a significant influence on the resistance reducing material seepage property, meaning that $b_{m}$ is variable but always larger than $b$, as shown in Figure 2.

The grounding resistance $R_{\text {electrode }}$ is equivalent to a series connection circuit of $R_{c 1}, R_{e 1}, R_{c 2}$, and $R_{e 2}$, where $R_{c 1}$ is the contact resistance between the resistance reducing material 
TABLE 1: Horizontal grounding electrode grounding resistance models.

\begin{tabular}{lc}
\hline Model & Formula \\
\hline Rudenberg, Zingraff & $R=\frac{1}{\sigma} \frac{1}{2 \pi L} \ln \frac{2 L}{d}\left(1+\frac{\ln (L / 2 h)}{\ln (2 L / d)}\right)$ \\
Tagg, Dwight & $R=\frac{1}{\sigma} \frac{1}{2 \pi L}\left(\ln \frac{4 L}{d}+\ln \frac{L}{h}-2+\frac{2 h}{L}-\frac{h^{2}}{L^{2}}+\frac{h^{4}}{8 L^{4}}\right)$ \\
Sunde, Schwarz & $R=\frac{1}{\sigma} \frac{1}{\pi L}\left(\ln \frac{2 L}{\sqrt{d h}}-1\right)$ \\
Neutral-point potential method & $R=\frac{1}{\sigma} \frac{1}{\pi L} \ln \frac{2 L}{\sqrt{d h}}$ \\
Average potential method $(h=0)$ & $R=\frac{1}{\sigma} \frac{1}{\pi L}\left(\ln \frac{4 L}{d}-1\right)$ \\
Neutral-point potential method $(h=0)$ & $R=\frac{1}{\sigma} \frac{1}{\pi L} \ln \frac{2 L}{d}$
\end{tabular}

TABLE 2: Functional relationships between $\eta$ and $\alpha$.

\begin{tabular}{lc}
\hline $\begin{array}{l}\text { Numbers of } \\
\text { electrodes }\end{array}$ & Utilization factor $\eta$ \\
\hline 2 & $\frac{1}{1+\alpha}$ \\
3 & $\frac{6-7 \alpha}{6+3 \alpha-12 \alpha^{2}}$ \\
4 & $\frac{12-10 \alpha}{12+16 \alpha-23 \alpha^{2}}$ \\
5 & $\frac{360-654 \alpha+241 \alpha^{2}}{360+270 \alpha-1495 \alpha^{2}+690 \alpha^{3}}$ \\
6 & $\frac{2160-2952 \alpha+555 \alpha^{2}}{2160+3312 \alpha-8439 \alpha^{2}+2047 \alpha^{3}}$ \\
\hline
\end{tabular}

and the electrode, $R_{e 1}$ is the resistance reducing material current divergence impedance, $R_{c 2}$ is the contact resistance between the resistance reducing material and the earth, and $R_{e 2}$ is the earth current divergence impedance:

$$
R_{\text {electrode }}=R_{c 1}+R_{e 1}+R_{c 2}+R_{e 2} \text {. }
$$

$R_{e 1}$ can be obtained by using

$$
R_{e 1}=\frac{1}{\sigma_{s}} \frac{1}{\pi L} \ln \frac{b_{m}}{a} .
$$

It can be simplified by considering the semicylinder for the resistance reducing material and the current divergence impedance. $R_{e 2}$ of a horizontal semicylindrical electrode can be derived from a model of a vertical electrode grounding impedance and the principle of mirror image; that is,

$$
R_{e 2}=\frac{1}{\sigma} \frac{1}{\pi L} \ln \frac{L}{b_{m}} .
$$

It can be seen that the resistance of the horizontal semicylindrical grounding electrode buried under the subsurface of the earth is equal to that derived by the midpoint potential method. The resistance reducing material has a gel-like property that keeps it in a certain shape and makes good

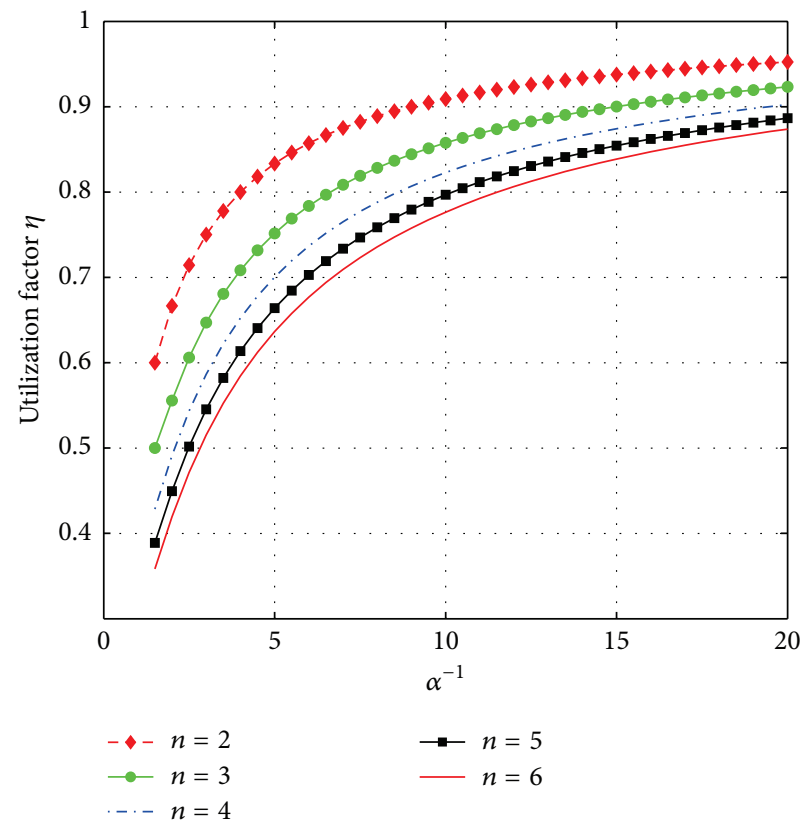

Figure 1: Variation of utilization factor $\eta$ with $\alpha^{-1}$ under $n$ dipole antennas.

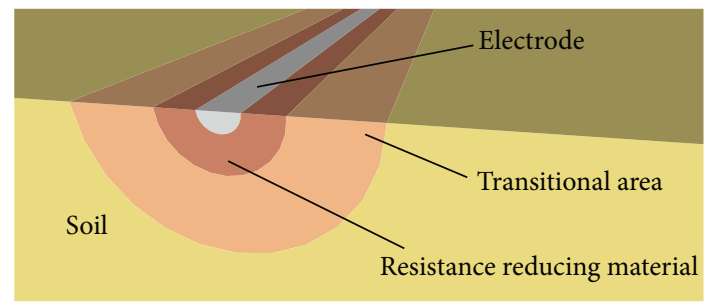

FIGURE 2: Grounding electrodes surrounded by resistance reducing material with high conductivity.

electrical contact with the medium surface. Therefore, the impedances $R_{c 1}$ and $R_{c 2}$ can be negligible.

For demonstration purposes, it can be assumed that the conductivity of the moist soil is $0.05 \mathrm{~S} / \mathrm{m}$, and the 
TABLE 3: Grounding impedance of electrodes and comparison between practical and theoretical utilization factor.

\begin{tabular}{|c|c|c|c|c|c|c|c|}
\hline $\begin{array}{l}\text { Electrodes } \\
\text { configuration }\end{array}$ & Interval $m$ & $\begin{array}{l}\text { Number of } \\
\text { electrodes }\end{array}$ & $\alpha$ & $\begin{array}{c}\text { Measured } \\
\text { impedance } \Omega\end{array}$ & $\tilde{\eta}$ & $\eta$ & $\begin{array}{c}\text { Utilization } \\
\text { factor deviation }\end{array}$ \\
\hline \multirow{9}{*}{ A } & 0.5 & 1 & 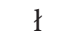 & 21.00 & 1 & 1 & 1 \\
\hline & 0.5 & 2 & 1 & 15.30 & 1 & 1 & 1 \\
\hline & 0.5 & 3 & 0.46 & 11.82 & 0.59 & 0.58 & $2.8 \%$ \\
\hline & 0.5 & 4 & 0.46 & 10.65 & 0.49 & 0.51 & $-3.7 \%$ \\
\hline & 0.5 & 5 & 0.46 & 9.36 & 0.45 & 0.47 & $-4.6 \%$ \\
\hline & 1.0 & 2 & 0.23 & 13.14 & 0.80 & 0.81 & $-1.2 \%$ \\
\hline & 1.0 & 3 & 0.23 & 10.20 & 0.69 & 0.72 & $-4.2 \%$ \\
\hline & 1.5 & 2 & 0.15 & 12.83 & 0.82 & 0.86 & $-4.7 \%$ \\
\hline & 2.0 & 2 & 0.12 & 12.39 & 0.85 & 0.89 & $-4.5 \%$ \\
\hline \multirow{4}{*}{ B } & 1.7 & 1 & 1 & 21.0 & 1 & 1 & 1 \\
\hline & 1.7 & 2 & 1 & 13.11 & 1 & 1 & 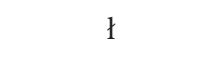 \\
\hline & 1.7 & 3 & 0.25 & 9.57 & 0.73 & 0.71 & $2.8 \%$ \\
\hline & 3.4 & 2 & 0.13 & 11.94 & 0.88 & 0.89 & $-1.1 \%$ \\
\hline
\end{tabular}

conductivity of the resistance reducing material is $2 \mathrm{~S} / \mathrm{m}$. The length of each grounding electrode $L$ is $1.2 \mathrm{~m}$ with radius $a=0.035 \mathrm{~m}$ and a grounding hemisphere equivalent radius of $r=0.17 \mathrm{~m}$. The earth current divergence impedance is $18.6 \Omega$ when the horizontal grounding electrodes are buried at the subsurface of the earth directly without the resistance reducing material. If the horizontal grounding electrodes are buried in semicylindrical pits of radius $b=0.2 \mathrm{~m}$ that are filled with resistance reducing material, which gives their equivalent radius $b_{m}>b$, in this case, we obtain $R_{e 1}=$ $0.25 \Omega$ and $R_{e 2}<9.5 \Omega$, meaning that the earth current divergence impedance $R_{\text {electrode }}<9.75 \Omega$. It can be seen that the grounding impedance would be effectively reduced by filling the pit with the resistance reducing material. In order to reduce the grounding impedance to $9.75 \Omega$ by using the grounding method without the resistance reducing material, there should be at least two electrodes placed parallelly with 3.4 m electrode interval, which would be an inefficient use of space and electrode material.

\subsection{Grounding Impedance Physical Experiment Simulation} and Verification. One set of experiments has been carried out on a sandy beach in Harbin. A stainless steel tube has been used as the test electrode, with length $1.2 \mathrm{~m}$ and diameter $0.14 \mathrm{~m}$. An Agilent U1733C LCR meter has been used to measure the impedance.

There are two configurations for placing the antennas: several electrodes are placed in parallel and the distance between any two contiguous electrodes is $D$ (configuration A), and the electrodes are placed in parallel in a head to tail configuration (configuration B).

The measurement results of the electrodes placed in configuration A and configuration B are shown in Table 3. According to the utilization factor expression in Table 2, the radius ratio can be written as $\alpha=2 R_{L} / R_{d}-1$ when $n=2$. The radius ratio $\alpha_{0}$ is 0.46 with the electrode interval $0.5 \mathrm{~m}$ in configuration A when $R_{L}=15.30 \Omega$ and $R_{d}=21.00 \Omega$. For the electrode intervals $1 \mathrm{~m}, 1.5 \mathrm{~m}$, and $2 \mathrm{~m}$, the radius ratios are $\alpha_{0} / 2, \alpha_{0} / 3$, and $\alpha_{0} / 4$. The radius ratio $\alpha_{1}$ is 0.25 in configuration B when $R_{L}=13.11 \Omega$. It is $\alpha_{1} / 2$ when the interval is $1.7 \mathrm{~m}$. Substituting radius ratios into utilization factor models in Table 2, the theoretical utilization factor $\eta$ can be derived. We can obtain the practical utilization factor $\tilde{\eta}$ based on (1).

The theoretical utilization factor matches the practical utilization factor well within a $\pm 5 \%$ deviation range. Compared with the electrodes placed in parallel, the grounding impedance of the electrodes arranged in parallel in a head to tail configuration is much smaller when they have the same gap. The arrangement of electrodes in parallel in a head to tail configuration increases the distance between each electrode because of its length, causing different grounding impedance. For instance, the interval in configuration $\mathrm{A}$ is $0.5 \mathrm{~m}$ with the gap $0.5 \mathrm{~m}$ but $1.7 \mathrm{~m}$ in configuration $\mathrm{B}$, accounting $1.2 \mathrm{~m}$ in length of the electrode itself, and the grounding impedance is $11.82 \Omega$ in configuration $A$ and $9.57 \Omega$ in configuration $B$. It has been proven that an impedance model of the horizontal grounding electrodes buried at the subsurface of the earth could be similarly calculated using the hemisphere grounding model. The electrodes arranged in parallel in a head to tail configuration are more suitable for applications such as in mines where there is a width restriction.

The experiment to optimize the antenna has been investigated at Xinqiang Mine in Qitaihe, Heilongjiang. The electrodes are buried in semicylindrical pits filled with resistance reducing material under the subsurface of the earth and in the mine and are parallelly arranged in a head to tail configuration. Each side of the antenna is composed of three electrodes, which are $1.3 \mathrm{~m}$ in length, $0.07 \mathrm{~m}$ in diameter, and at an interval of $1.5 \mathrm{~m}$. The antennas are buried in pits that are $1.3 \mathrm{~m}$ in length and $0.3 \mathrm{~m}$ in diameter. The distance between two sides of the antenna on the ground is $40 \mathrm{~m}$ but $80 \mathrm{~m}$ within the mine. Moist clay is the predominant soil on the ground, which has a conductivity within the range of $0.01 \sim$ $0.05 \mathrm{~S} / \mathrm{m}$. The conductivity in mine is in the range of $0.005 \sim$ $0.02 \mathrm{~S} / \mathrm{m}$, due to the fact that the predominant soil is rock. 
TABLE 4: Grounding impedance of electrodes on the ground and in the mine.

\begin{tabular}{lcc}
\hline $\begin{array}{l}\text { Frequency } \\
\mathrm{kHz}\end{array}$ & $\begin{array}{c}\text { Grounding } \\
\text { impedance of } \\
\text { electrodes on the } \\
\text { ground } \Omega\end{array}$ & $\begin{array}{c}\text { Grounding } \\
\text { impedance of } \\
\text { electrodes in the mine } \\
\Omega\end{array}$ \\
\hline 0.1 & 5.4 & 12.7 \\
1 & 4.5 & 14.0 \\
10 & 4.4 & 13.9 \\
\hline
\end{tabular}

The impedance of each antenna is measured by an Agilent U1733C LCR meter, and the results are shown in Table 4.

As seen in Table 4, the impedance of the dipole antenna on the ground is about $5 \Omega$, indicating that the grounding impedance of each side of the antenna is less than $2.5 \Omega$. The conductivity in the mine tunnel is lower than that on the ground as the geological material within the mine is mainly rock. The impedance of the dipole antenna in the mine is $13 \Omega$ and $6.5 \Omega$ at each side of the antenna.

The output current ability of the TTE communication system and its range can be improved by placing the antennas in parallel in a head to tail configuration and filling the pits with resistance reducing material. This grounding model meets the requirement to reduce the underground communication systems' power.

\section{Propagation of Dipole Antennas in a Semi-Infinite Layered Conducting Medium}

3.1. Simulation Model of Dipole Antenna in Layered Conducting Medium. A numerical calculation of the electric field has been employed successfully in many applications, but it cannot describe the propagation successfully for complicated structures, such as layered conducting mediums [15]. To investigate the propagation of dipole antenna in layered conducting medium, the full-wave electromagnetic simulation model with the CST STUDIO SUIT electromagnetic tool is set up to accurately investigate the propagation.

According to a mine regional stratigraphic structural table, the Quaternary, Cretaceous, and Jurassic compositions are the main structural components of mines. The surface is a Quaternary system composed of alluvial sand. The middle layer is a Cretaceous system dominated by purple sandstone and grey-greenish mudstone on the upper layer and purplish-red conglomerate with mudstone in the lower layer. The bottom layer is a Jurassic system, with greygreenish sandstone, conglomerate, mudstone, and marl on the upper level, volcanic rock, rhyolite, and mudstone in the middle level, and sandstone and conglomerate in the lower level. In order to reduce the influence of boundary conditions, the thickness of the air on the ground is set at $50 \mathrm{~m}$, and the simulation area is $2000 \mathrm{~m}$ in length and width. The electrical parameters of these strata are shown in Table 5.

The full-wave electromagnetic simulation results with the CST STUDIO SUIT electromagnetic tool are presented with a moment $p=100 \mathrm{~A} \cdot \mathrm{m}$ on the dipole antenna. To understand the full-wave propagation characteristics of the dipole in the
TABLE 5: The electrical parameters of strata.

\begin{tabular}{llcc}
\hline Strata & Attribute & $\begin{array}{c}\text { Conductivity } \\
\text { S/m }\end{array}$ & $\begin{array}{c}\text { Thickness } \\
\mathrm{m}\end{array}$ \\
\hline Quaternary & Alluvial sand, clay & 0.01 & 20 \\
\hline \multirow{5}{*}{ Cretaceous } & Sandstone & 0.006 & 180 \\
& Coal & 0.01 & 2 \\
& Conglomerate & 0.01 & 200 \\
& Coal & 0.01 & 3 \\
& Conglomerate & & \\
& mixed with & 0.008 & 160 \\
& mudstone & & \\
\hline \multirow{5}{*}{ Jurassic } & Marl & 0.003 & 260 \\
& Volcanic rock & 0.001 & 240 \\
& Conglomerate & 0.01 & 200 \\
\hline
\end{tabular}

TABLE 6: Electric field of the field point.

\begin{tabular}{lccc}
\hline $\begin{array}{l}\text { Frequency } \\
\mathrm{kHz}\end{array}$ & $\begin{array}{c}\text { Field strength at } \\
-200 \mathrm{~m} \mu \mathrm{V} / \mathrm{m}\end{array}$ & $\begin{array}{c}\text { Field strength at } \\
-300 \mathrm{~m} \mu \mathrm{V} / \mathrm{m}\end{array}$ & $\begin{array}{c}\text { Field strength at } \\
-600 \mathrm{~m} \mu \mathrm{V} / \mathrm{m}\end{array}$ \\
\hline 0.1 & 174.0 & 56.0 & 8.8 \\
0.3 & 187.5 & 65.2 & 12.2 \\
1 & 204.3 & 66.6 & 9.0 \\
1.5 & 204.5 & 61.9 & 6.3 \\
2 & 199.7 & 56.2 & 4.5 \\
3 & 183.7 & 45.3 & 2.4 \\
5 & 147.4 & 29.0 & 0.84 \\
10 & 81.8 & 10.4 & 0.096 \\
20 & 28.2 & 1.9 & 0.003 \\
30 & 11.2 & 0.5 & 0.0002 \\
\hline$f_{M} \mathrm{kHz}$ & 1.5 & 1.0 & 0.3 \\
$-3 \mathrm{~dB}$ & & & \\
bandwidth & 5.0 & 3.0 & 1.0 \\
$\mathrm{kHz}$ & & & \\
\hline
\end{tabular}

layered conducting earth, the dipole antenna working at operating frequency $1.5 \mathrm{kHz}$ is simulated and shown in Figure 3. It is found that there is a huge change at the interface between every two mediums, making the propagation complicated. As the points investigated are far enough from the boundary, the boundary condition has little influence on the simulation results. The full-wave electromagnetic simulation results of the field point at positions of $-200 \mathrm{~m},-300 \mathrm{~m}$, and $-600 \mathrm{~m}$ are shown in Table 6.

As shown in the simulation results, there is an optimum frequency $f_{M}$ at each propagation depth. For the field point at position $-200 \mathrm{~m}, f_{M}$ is $1.5 \mathrm{kHz}$ with a $-3 \mathrm{~dB}$ bandwidth $5 \mathrm{kHz}$. When the field point is located at position $-300 \mathrm{~m}$, $f_{M}=1.0 \mathrm{kHz}$ and the $-3 \mathrm{~dB}$ bandwidth decreases to $3 \mathrm{kHz}$. For the field point at $-600 \mathrm{~m}, f_{M}$ is $0.3 \mathrm{kHz}$ and the $-3 \mathrm{~dB}$ bandwidth is $1 \mathrm{kHz}$. In this instance, $f_{M}$ and the $-3 \mathrm{~dB}$ bandwidth decrease as the distance between the source point and the field point increases. For extremely low frequency, the electric field at the field point shows characteristic of a static 


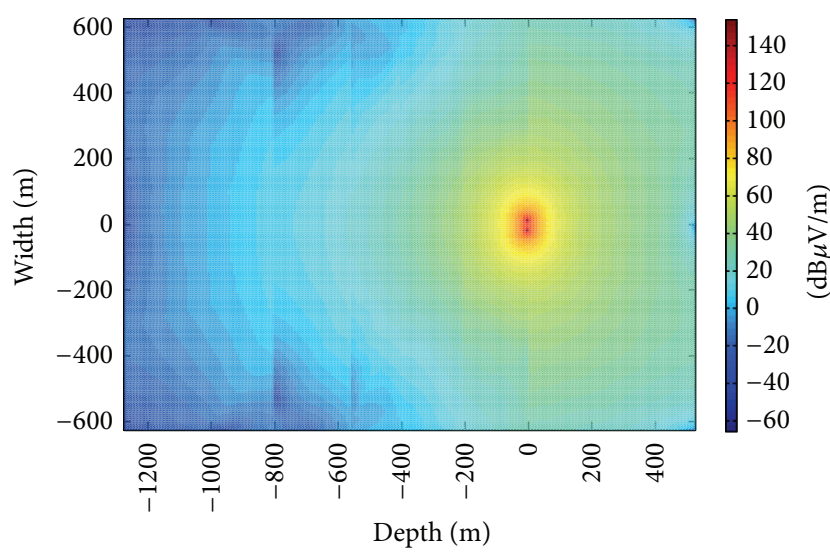

FIgURE 3: Propagation of the dipole in layered conducting earth at operating frequency $1.5 \mathrm{kHz}$.

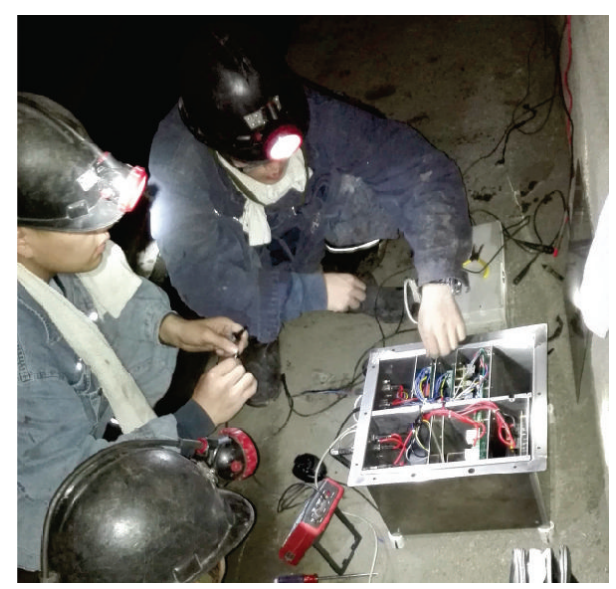

FIGURE 4: TTE communication experiment in the mine.

field, and the bandwidth lower than $f_{M}$ is within the $-3 \mathrm{~dB}$ bandwidth. A further simulation result has concluded that when the communication distance is within a range of 200 $600 \mathrm{~m}$ vertically and the conductivity of the earth is within $0.001 \sim 0.05 \mathrm{~S} / \mathrm{m}$, the most favorable operating frequencies are below $10 \mathrm{kHz}$.

\subsection{Field Experiment for the Propagation of a Dipole Antenna.} As shown in Figure 4, a set of experiments has been carried out at Xinqiang Mine in Heilongjiang Province. The test point at the mine is $300 \mathrm{~m}$ from the test point on the ground vertically and $300 \mathrm{~m}$ in straight-line distance from the wellhead horizontally. The test point is not connected with the lift shaft directly so that the electromagnetic energy cannot propagate along that. The interelectrode distance for the antenna in the mine is $80 \mathrm{~m}$ and for the antenna on the ground is $40 \mathrm{~m}$. The dipole antenna on the ground is parallel to that in the mine, with their azimuth at $260^{\circ}$.

For the propagation measurement, the signal source, power amplifier, low noise linear amplitude to amplify the voltage on the dipole antenna, and scope to store the wave data are used. The power signal output by the power amplifier

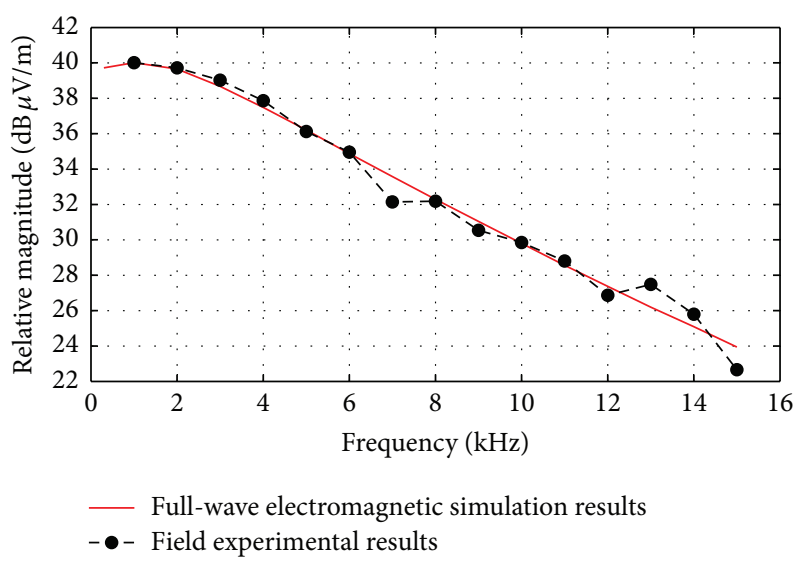

FIgURE 5: The full-wave electromagnetic simulation results and the field experimental results curve.

is loaded onto the dipole antenna, with an operating frequency in the range of $1 \sim 15 \mathrm{kHz}$. The power stage and the dipole antenna are connected by a multifiber copper wire with a length in the range of $20 \sim 40 \mathrm{~m}$. There is an additional inductive-resistive component on the total impedance. Therefore, the power amplifier output should be regulated so that the moment on the antenna is a fixed value of $p=$ $100 \mathrm{~A} \cdot \mathrm{m}$.

The full-wave electromagnetic simulation model with the CST STUDIO SUIT electromagnetic tool is set, where the thickness of the air on the ground is $50 \mathrm{~m}$, and the simulation area is $2000 \mathrm{~m}$ in length and width. The upper layer of the earth is alluvial sand and sandstone with the thickness of about $180 \mathrm{~m}$ and conductivity $0.007 \mathrm{~S} / \mathrm{m}$. The next layer is conglomerate and coal with the thickness of about $200 \mathrm{~m}$ and conductivity $0.005 \mathrm{~S} / \mathrm{m}$. The bottom layer is mudstone with the thickness of about $160 \mathrm{~m}$ and conductivity $0.008 \mathrm{~S} / \mathrm{m}$. Figure 5 shows a comparison of the relative magnitude of the full-wave electromagnetic simulation results and field experimental results.

From Figure 5, the magnitude decreases as the frequency increases, with a slope of about $-1.2 \mathrm{~dB} / \mathrm{kHz}$ for the frequency higher than $3 \mathrm{kHz}$, but for lower frequency the slope tends to be stable. It can be seen from Table 7 that most of the linear errors between the simulation results and the experimental results are less than $6 \%$. Four of the linear errors deviate from normal value because of the tone interference at certain frequency. But the experimental results are close to the simulation results in Figure 5, indicating that the electromagnetic tool can represent the propagation of a dipole antenna in a layered conducting medium accurately. For the mine studied, the optimal frequency at a depth of $-300 \mathrm{~m}$ is $1 \mathrm{kHz}$.

\section{TTE Communication for Mine}

A TTE communication system for mines has been designed, which operates at frequencies of $2 \mathrm{kHz}$ or $8 \mathrm{kHz}$, with a message data rate of $25 \mathrm{bps}$ and $50 \mathrm{bps}$ for digital voice information. It works in the half duplex, using 2DPSK modulation and demodulation technology. A high-performance speech 
TABLE 7: The full region electromagnetic simulation results and the field experimental results.

\begin{tabular}{lccc}
\hline $\begin{array}{l}\text { Frequency } \\
\mathrm{kHz}\end{array}$ & $\begin{array}{c}\text { Simulation results } \\
\mathrm{dB} \mu \mathrm{V} / \mathrm{m}\end{array}$ & $\begin{array}{c}\text { Experimental results } \\
\mathrm{dB} \mu \mathrm{V} / \mathrm{m}\end{array}$ & $\begin{array}{c}\text { Linear error } \\
\%\end{array}$ \\
\hline 0.3 & 39.72 & 1 & 1 \\
1 & 40.01 & 30.01 & 0.00 \\
2 & 39.65 & 39.02 & 0.81 \\
3 & 38.67 & 37.86 & 4.11 \\
4 & 37.48 & 36.12 & 4.47 \\
5 & 36.21 & 34.95 & -1.03 \\
6 & 34.87 & 32.14 & 0.93 \\
7 & 33.58 & 32.18 & -15.28 \\
8 & 32.29 & 30.54 & -1.26 \\
9 & 31.04 & 29.85 & -5.59 \\
10 & 29.80 & 28.80 & 0.58 \\
11 & 28.56 & 26.87 & 2.80 \\
12 & 27.37 & 27.48 & -5.59 \\
13 & 26.19 & 25.80 & 16.01 \\
14 & 25.09 & 22.66 & 8.52 \\
15 & 23.94 & & -13.70 \\
\hline
\end{tabular}

compression MDV chip is used, with an ultralow voice coding rate of 600 bps. Digital voice data are error correction coded, stored in a buffer, and then transmitted with a low data rate.

The TTE communication experiment has been performed in Xinqiang Coal Mine, Heilongjiang Province. A dipole antenna is placed in the pits with radius $0.2 \mathrm{~m}$ filled with a resistance reducing material. Each side of the antenna includes 3 electrodes that are $1.2 \mathrm{~m}$ in length and $0.7 \mathrm{~m}$ in diameter, parallelly arranged in a head to tail configuration, with a gap of $1 \mathrm{~m}$. The antenna impedance on the ground is reduced to $8 \Omega$, with a $40 \mathrm{~m}$ electrode interval. The impedance of the antenna in the mine is reduced to $13 \Omega$, with an $80 \mathrm{~m}$ electrode interval. Thus, the grounding impedance is effectively reduced compared with that in [3]. For an efficient transmitter, the transformer turn ratios $1.4: 1$ and $1.8: 1$ are fixed in order to match the ideal power stage load value $4 \Omega$ [3]. The vertical distance from the ground to the tunnel of the mine is $300 \mathrm{~m}$. The results show that the short message and digital voice can be effectively received at $8 \mathrm{kHz}$, when the output power is greater than $180 \mathrm{~W}$. However, the system output power is only $30 \mathrm{~W}$ at the operating frequency $2 \mathrm{kHz}$ with the same communication performance. Thus, the dipole antenna can be optimized using the optimal electric dipole antenna model and the communication output power can be reduced by transporting information at the carrier frequency $f_{M}$, which further proves the results in this paper.

\section{Conclusions}

In this paper, a grounding impedance model is presented for several horizontal electrodes placed in parallel. The analysis and contrast experiments have shown that the impedance of the dipole antenna on the ground is reduced to $5 \Omega$ and $13 \Omega$ in the mine. Compared with the grounding impedance $300 \Omega$ in [3], the optimal grounding impedance model can effectively reduce the grounding impedance.

The full-wave electromagnetic simulation model with the CST STUDIO SUIT electromagnetic tool can describe the propagation property of dipole in layered conducting medium and verified by field experiment. There is a single operating frequency for each field point at each depth of the layered earth that can achieve maximum field strength. The $f_{M}$ in the full-wave simulation model at position $-200 \mathrm{~m}$ is $1.5 \mathrm{kHz}, 1 \mathrm{kHz}$ at $-300 \mathrm{~m}$, and $0.3 \mathrm{kHz}$ at $-600 \mathrm{~m}$. The operating frequency decreases as the communication distance or the earth conductivity increases. Thus, TTE communication is suitable for short range communication in the vertical direction.

It is shown that the TTE communication method could be successfully used for communication across a distance of $300 \mathrm{~m}$ vertically from the ground into the mine, with an output power reduced from $180 \mathrm{~W}$ at $8 \mathrm{kHz}$ to $30 \mathrm{~W}$ at $2 \mathrm{kHz}$ and an information data rate of 50 bps. The communication system can be placed in a fixed position and can be used for transmitting low data rate information, such as monitoring information or a distress signal in emergency situation.

\section{Competing Interests}

The authors declare that they have no competing interests.

\section{Acknowledgments}

This research has been supported by International Science \& Technology Cooperation Program of China (2014 DFR10240), Harbin Science and Technology Research Projects (2013AE1BE003), Science Foundation of Heilongjiang Province QC2015075, and the Fundamental Research Funds for the Central Universities (China) [HEUCF1508].

\section{References}

[1] H. Wang, K. Zheng, K. Yang, and Y. Ma, "Electromagnetic field in air produced by a horizontal magnetic dipole immersed in sea: theoretical analysis and experimental results," IEEE Transactions on Antennase Propagation, vol. 62, no. 9, pp. 46474655, 2014.

[2] V. Bataller, A. Muñoz, P. M. Gaudó, A. Mediano, J. A. Cuchí, and J. L. Villarroel, "Earth impedance model for through-the-earth communication applications with electrodes," Radio Science, vol. 45, no. 6, Article ID RS6015, pp. 2970-2980, 2010.

[3] V. Bataller, A. Muñoz, P. M. Gaudó, A. Mediano, J. A. Cuchí, and J. L. Villarroel, "Improving medium access in through-the-earth VLF-LF communications," Journal of Communications, vol. 4, no. 4, pp. 284-294, 2009.

[4] A. A. Abdou, A. Shaw, A. Mason, A. Al-Shamma’a, J. Cullen, and S. Wylie, "Electromagnetic (EM) wave propagation for the development of an underwater Wireless Sensor Network (WSN)," in Proceedings of the IEEE Sensors, pp. 1571-1574, IEEE, Limerick, Ireland, October 2011.

[5] G. Adw, Channel Characterisation and System Design for Sub Surface Communications, University of Leeds, Leeds, UK, 2003. 
[6] M. B. Kraichman, Handbook of Electromagnetic Propagation in Conducting Media, University of Michigan Library, 1970.

[7] U. M. Cella, R. Johnstone, and N. Shuley, "Electromagnetic wave wireless communication in shallow water coastal environment: theoretical analysis and experimental results," in Proceedings of the 4th ACM International Workshop on UnderWater Networks (WUWNet '09), pp. 1-8, Berkeley, Calif, USA, November 2009.

[8] P. R. Bannister, "Quasi-static fields of dipole antennas at the earth's surface," Radio Science, vol. 1, no. 11, pp. 1321-1332, 1966.

[9] Y. H. Xu, K. Li, and L. Liu, "Electromagnetic field of a horizontal electric dipole in the presence of a four-layered region," Progress in Electromagnetics Research, vol. 81, pp. 371-391, 2008.

[10] C. Liu, L.-G. Zheng, and Y.-P. Li, "Study of ELF electromagnetic fields from a submerged horizontal electric dipole positioned in a sea of finite depth," in Proceedings of the 3rd IEEE International Symposium on Microwave, Antenna, Propagation and EMC Technologies for Wireless Communications (MAPE '09), pp. 152157, Beijing, China, October 2009.

[11] S. Bourg, B. Sacepe, and T. Debu, "Deep earth electrodes in highly resistive ground: frequency behaviour," in Proceedings of the IEEE International Symposium on Electromagnetic Compatibility, pp. 584-589, Atlanta, Ga, USA, August 1995.

[12] J. He and R. Zeng, Power System Grounding, Science Press, 2007.

[13] K. S. Lee and Y. K. Chung, "Apparatus and method for calculating length of carbon grounding electrode module based on twolayered distributed constant circuit," Patent WO 2012050338 A3, 2013.

[14] Z. Feng, L. Lu, and J. Feng, "Research on reducing grounding resistance of transmission line tower grounding grid," in Proceedings of the 2nd Annual Conference on Electrical and Control Engineering (ICECE '11), pp. 1216-1219, Yichang, China, September 2011.

[15] K. Li, Fields and Waves in Layered Media, Zhejiang University Press, Zhejiang, China, 2010. 


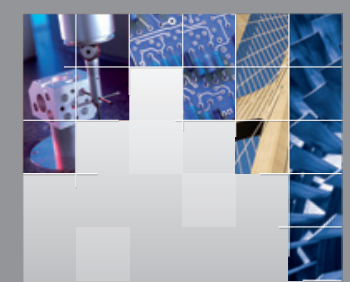

\section{Enfincering}
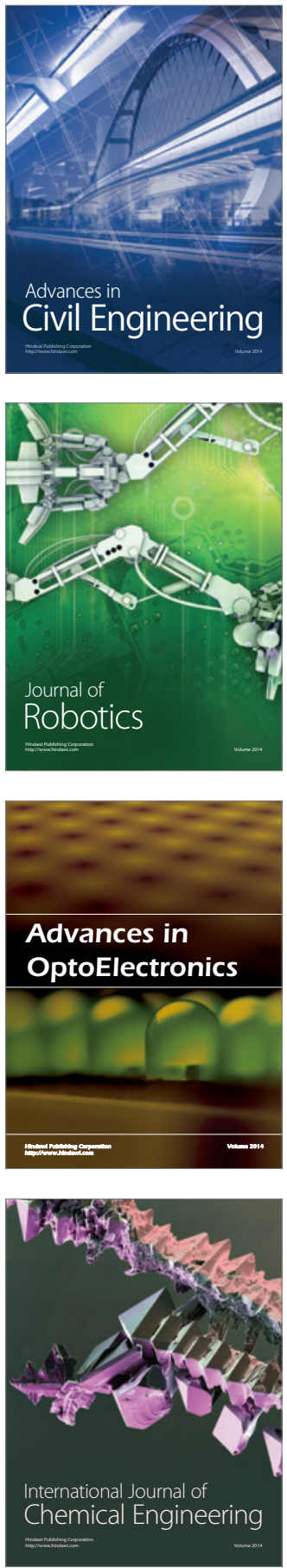

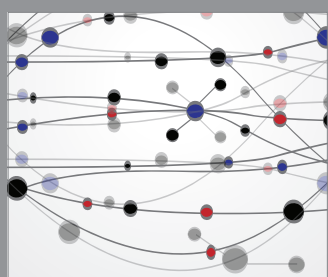

The Scientific World Journal

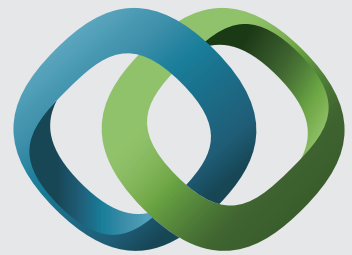

\section{Hindawi}

Submit your manuscripts at

http://www.hindawi.com
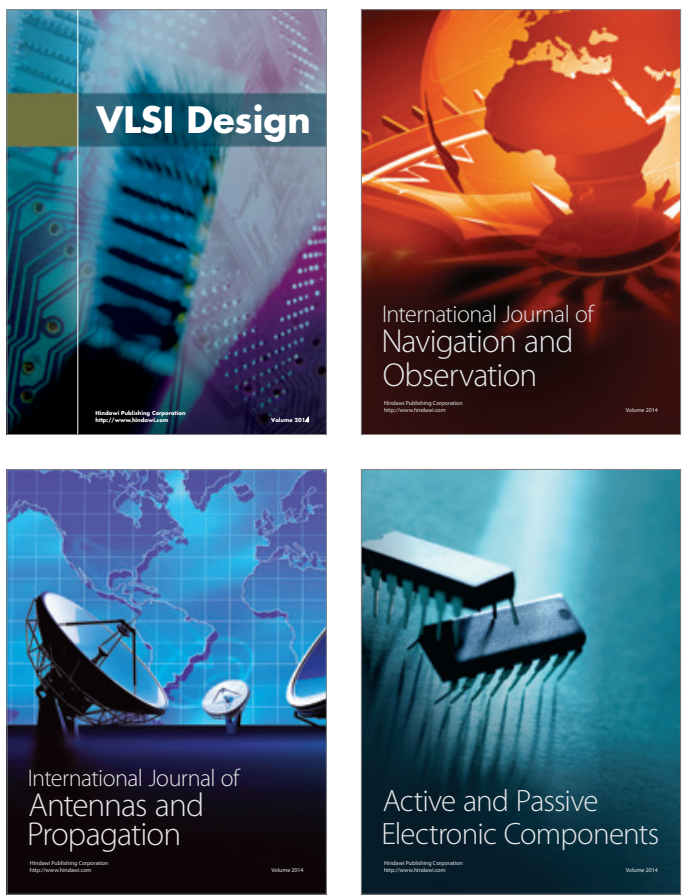
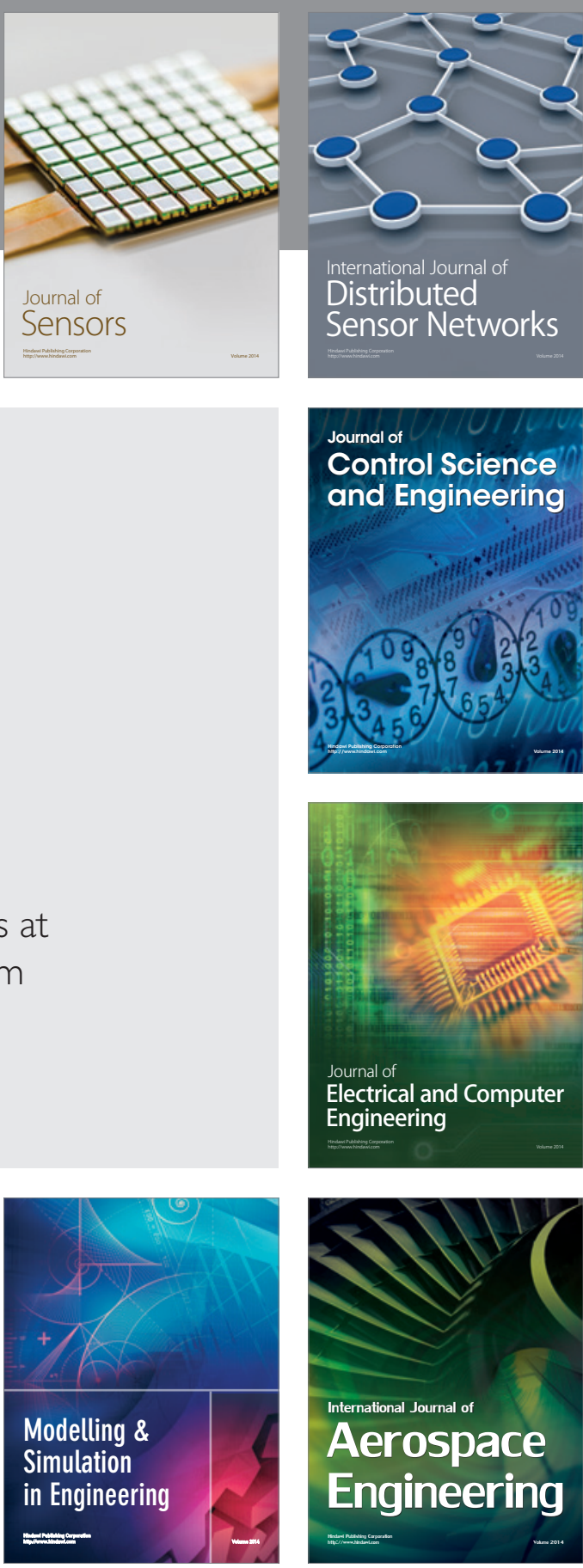

International Journal of

Distributed

Sensor Networks

Journal of

Control Science

and Engineering
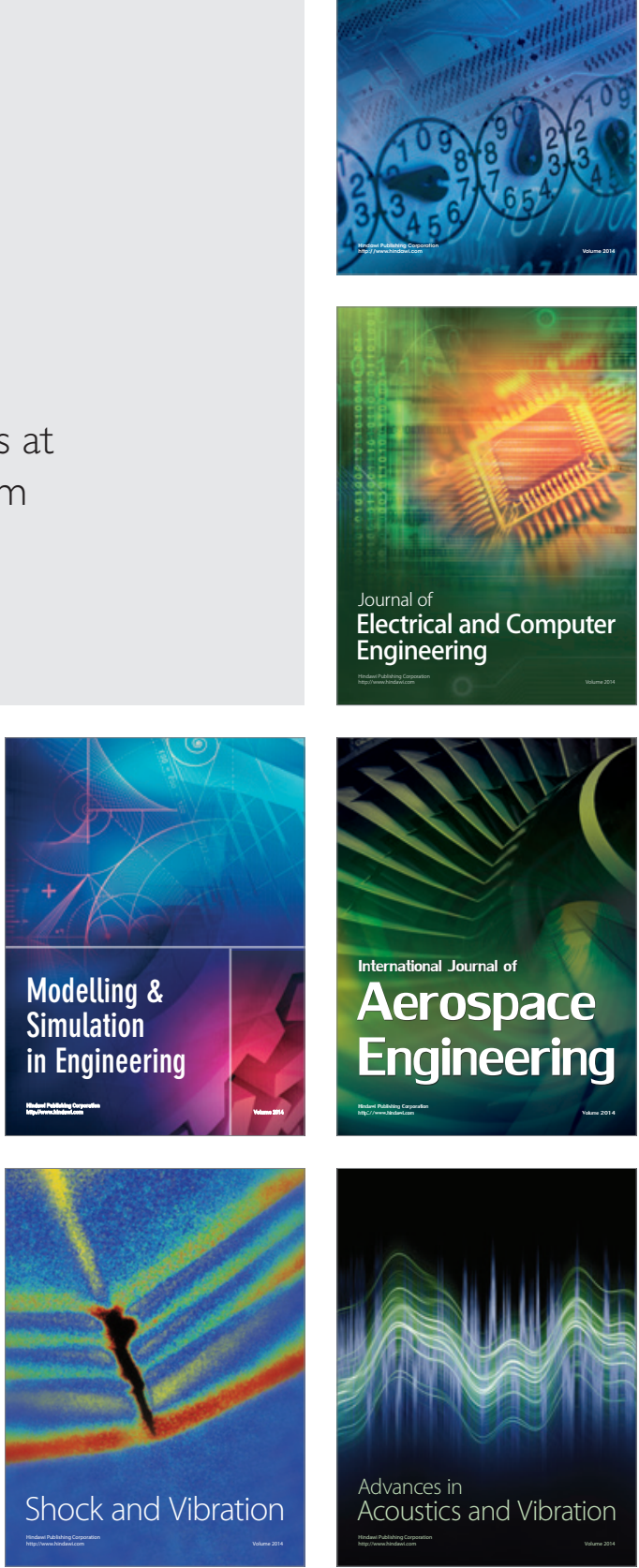